\title{
EMT related circular RNA expression profiles identify circSCYL2 as a novel molecule in breast tumor metastasis
}

\author{
CHUNLEI YUAN $^{1}$, XULIANG LUO $^{2}$, XIANG ZHAN $^{3}$, HUIHUI ZENG $^{3}$ and SIJIA DUAN ${ }^{1}$ \\ ${ }^{1}$ Department of Breast Surgery, The Second Affiliated Hospital of Nanchang University; \\ ${ }^{2}$ Medical College of Nanchang University, Nanchang, Jiangxi 330000; ${ }^{3}$ Department of General Surgery, \\ The People's Hospital of Le 'An County, Fuzhou, Jiangxi 344000, P.R. China
}

Received September 20, 2019; Accepted February 14, 2020

DOI: $10.3892 /$ ijmm.2020.4550

\begin{abstract}
Substantial evidence indicates that circular RNAs (circRNAs) play vital roles in several diseases, especially in cancer development. However, the functions of circRNAs in breast cancer metastasis remain to be investigated. This study aimed to identify the key circRNAs involved in epithelial mesenchymal transition (EMT) of breast cancer and evaluated their molecular function and roles in pathways that may be associated with tumor metastasis. An EMT model was constructed by treating breast cancer cells MCF-7 and MDA-MB-231 with transforming growth factor- $\beta 1$. High-throughput RNA sequencing was used to identify the differentially expressed circRNAs in EMT and blank groups of two cells, and reverse transcription-quantitative PCR was used to validate the expression of circSCYL2 in human breast cancer tissues and cells. The effects of circSCYL2 on breast cancer cells were explored by transfecting with plasmids and the biological roles were assessed using transwell assays. EMT groups of breast cancer cells exhibited the characteristics of mesenchymal cells. Furthermore, the present study found that 7 circRNAs were significantly upregulated in both the MCF-7 EMT and MDA-MB-231 EMT groups, while 16 circRNAs were significantly downregulated. The current study identified that circSCYL2 was downregulated in breast cancer tissues and cell lines, and that circSCYL2 overexpression inhibited cell migration and invasion. This study provides expression profiles of circRNAs in EMT groups of breast cancer cells. circSCYL2, which is downregulated in breast cancer tissues and cells, may play an important role in breast cancer EMT progression.
\end{abstract}

Correspondence to: Dr Sijia Duan, Department of Breast Surgery, The Second Affiliated Hospital of Nanchang University, 1 Mingde Road, Donghu, Nanchang, Jiangxi 330000, P.R. China

E-mail: duansj85@163.com

Key words: breast cancer, transforming growth factor- $\beta 1$, circRNA, circSCYL2, epithelial mesenchymal transition

\section{Introduction}

Breast cancer is a malignant tumor that occurs in the epithelial tissue of the breast glands and is the most frequently occurring cancer in women all over the world (1). The global incidence of breast cancer has been on the rise since the late 1970s and breast cancer has become a major public health problem in current society. Despite the improved prognosis of patients with breast cancer resulting from early diagnosis, radical surgery, chemotherapy, radiotherapy, hormonal therapy and the development of targeted therapy, breast cancer remains the leading cause of cancer mortality in women $(2,3)$. The high mortality rates in breast cancer patients are associated with recurrence, drug resistance and the high frequency of metastasis (4). In most cases, patients with metastasis are not eligible for surgery and chemotherapy or radiotherapy, which do not contribute significantly to cure them (5-7). Metastasis remains a major obstacle in achieving a radical cure and preventing death of breast cancer patients. Therefore, it is essential to understand the molecular pathways involved in causing metastasis of breast cancer.

Tumor metastatic invasiveness is linked with the increased migration capacity of epithelial cells. This process is known as the epithelial-mesenchymal transition (EMT) (8). Epithelial cancer cells in the lactiferous duct having undergone EMT are capable of undergoing metastasis and are more invasive (9-11). Transforming growth factor (TGF)- $\beta 1$ has been reported to induce EMT (12). The loss of epithelial (E)-cadherin, an adherens junction cell surface protein expressed in epithelial cells is the principal characteristic of EMT. Vimentin, which is the major cytoskeletal component of mesenchymal cells was increased significantly by the treatment of TGF- $\beta 1$ (13). In breast cancer, EMT both elevates the migratory capacity and invasive potential of tumor cells and initiates pro-tumorigenic alterations in the tumor microenvironment (14). CORM-A1 and DETA/NO combination therapy showed antimetastatic action and resulted in normalization of endothelial metabolism, diminution of platelet activation, and inhibition of EMT progression (12). Coenzyme $\mathrm{Q}_{0}$ attributed to the phosphatidyl inositol 3 kinase $(\mathrm{PI} 3 \mathrm{~K}) /$ protein kinase $\mathrm{B}(\mathrm{AKT}) /$ nuclear factor $(\mathrm{NF}) \kappa \mathrm{B} /$ matrix metalloproteinase-9 signaling pathway through reactive oxygen species-mediated apoptosis can inhibit the progression of metastasis as well as EMT (in vitro 
and in vivo) (15). Metastasis of breast cancer remains challenging for the current clinical treatments and more than half of the patients suffer from a disease relapse due to distant metastases (16). Therefore, further research into the underlying molecular mechanism and development of more targets of EMT and metastasis of breast cancer is urgent.

Circular RNA (circRNA) is a novel type of endogenous non-coding RNA without a 5' cap and 3' poly (A) end. They are widely expressed in the central nervous system and enrich biological functions as demonstrated by some reports such as acting as miRNA sponges, regulating transcription or binding to the miRNA binding proteins $(17,18)$. Previously, growing evidence has confirmed that circRNAs are associated with multiform cancerous biological progression $(19,20)$, and an increasing number of functional circRNAs are discovered in breast cancer. For instance, targeting the circBMPR2/microRNA (miR)-553/USP4 axis can be used as a potential therapeutic approach for breast cancer; circ_0103552 predicts dismal prognosis and promotes breast cancer cell proliferation and invasion by sponging miR-1236 $(21,22)$; hsa circ_001569 can facilitate metastasis through the modulation of the PI3K-AKT pathway, which is an unfavorable prognostic factor of breast cancer (23). However, little is known about the global change of circRNA profiles during EMT, which is an important step in tumor metastasis.

In this study, expression profiles of circRNAs in breast cancer cells with TGF- $\beta 1$ induction were analyzed to confirm the EMT related circRNAs, suggesting that the different expression levels of circRNAs may be closely related to breast cancer metastasis. This study validated circSCYL2, which could suppress breast cancer cell migration and invasion, may serve as biomarkers during tumor metastasis. The present study aimed to explore the functions of circRNAs and furnish novel therapeutic targets for tumor metastasis.

\section{Materials and methods}

Cell culture and establishment of EMT model. The human breast cancer cell lines MCF-7 and MDA-MB-231 used in this research were obtained from Yingbio Technology. MCF-7 cells were incubated in Dulbecco's modified Eagle's medium (10-013-CVR; Corning, Inc.) with 10\% (v/v) heat-inactivated fetal bovine serum (FBS; Gibco; Thermo Fisher Scientific, Inc.) and $1 \%$ penicillin-streptomycin (P/S, E607011; Sangon Biotech Co., Ltd.) and were cultured in a humidified $37^{\circ} \mathrm{C}$ incubator with $5 \% \mathrm{CO}_{2}$. While MDA-MB-231 cells were incubated in Leibovitz's 15 medium (L15, E600016-0500; BBI Solutions) with $10 \%$ (v/v) FBS and $1 \% \mathrm{P} / \mathrm{S}$ and were cultured in a humidified $37^{\circ} \mathrm{C}$ incubator with $100 \%$ air. These cells, which were incubated in medium with $0.5 \%$ FBS, were treated with $20 \mathrm{ng} / \mathrm{ml}$ TGF- $\beta 1$ (100-21-10; Peprotech, Inc.) for $48 \mathrm{~h}(24,25)$. The control cells were incubated in medium with $0.5 \%$ FBS and treated with DMSO. Then, cell pictures were captured by an inverted phase-contrast microscope (Shanghai Optical Sixth Factory). The cells treated by TGF- $\beta 1$ were named as cell-EMT and the control cells were named as cell-blank.

Western blotting. The total protein of cells (MCF-7-EMT, MCF-7-blank, MDA-MB-231-EMT and MDA-MB-231-blank) was extracted using RIPA Lysis Buffer (Thermo Fisher Scientific, Inc.) and the concentration was measured using a bicinchoninic acid assay kit. Total $150 \mu \mathrm{g}$ protein samples were separated via $10 \%$ SDS-PAGE and transferred to a polyvinylidene fluoride membrane (EMD Millipore). After blocking in $5 \%$ milk at room temperature for $3 \mathrm{~h}$, the membranes were incubated with primary antibodies against E-cadherin (cat. no. sc-52327; 1:500; Santa Cruz Biotechnology, Inc.), Vimentin (cat. no. ab8978; 1:500; Abcam) and GAPDH (cat. no. 60004-1-Ig; 1:1,000; Proteintech, Inc.) overnight at $4{ }^{\circ} \mathrm{C}$. This was followed by incubation with goat anti-mouse IgG-horseradish peroxidase antibody (cat. no. A0216; 1:1,000; Beyotime Institute of Biotechnology) at room temperature for $2 \mathrm{~h}$ and the blots were detected using the enhanced chemiluminescence reagent (Thermo Fisher Scientific, Inc.). Images were captured by a ChemiDoc Gel Imaging system (Thermo Fisher Scientific, Inc.) and optical density was analyzed by Image J software (version 1.8.0; National Institute of Health). Results represent the means of three independent experiments performed in triplicates. P-values were determined by using t-tests and $\mathrm{P}<0.05$ was considered to indicate a statistically significant difference.

RNA isolation, RNA library construction and sequencing. Total RNA from two paired samples (MC-7 EMT and MCF-7 blank, MDA-MB-231 EMT and MDA-MB-231 blank) was extracted using the TRIZOL reagent (Invitrogen; Thermo Fisher Scientific, Inc.), followed by an assessment for quantity and purity using the microspectrophotometer (TGem Tiangen Biotech Co., Ltd.). The RNA integrity number was analyzed using a UV Spectrophotometer (UV-2000; Tanon Science and Technology Co., Ltd.). Thereafter, $1 \mu \mathrm{g}$ total RNA from each sample was used to prepare the library according to the following procedures: Ribosomal RNAs (rRNAs) were removed from total RNA using an rRNA probe and RNase $\mathrm{H}$. The ribosomal-depleted RNA of target fragment size (300 bp) was obtained using metal ions, reverse transcribed into cDNA, connected with adapters, followed by fragment sorting, enrichment with PCR and then purified library products were quantified and evaluated using the Quant-iT ${ }^{\mathrm{TM}}$ PicoGreen $^{\circledR}$ dsDNA Assay kit (Beckman Coulter, Inc.) and Agilent 2200. The process of reverse transcription in RNA library construction was as follows: First-strand cDNA was synthesized by the reagents of Actinomycin D (Vazyme Biotech Co., Ltd.), first Strand Enzyme Mix (Vazyme Biotech Co., Ltd.), first Strand Buffer, dNTPs random primer using the following process at $25^{\circ} \mathrm{C}$ for $10 \mathrm{~min}, 42^{\circ} \mathrm{C}$ for $15 \mathrm{~min}$ and $75^{\circ} \mathrm{C}$ for $15 \mathrm{~min}$. Second Strand/End Repair Enzyme Mix, second Strand Marking Buffer and dNTPs were mixed at $16^{\circ} \mathrm{C}$ for $60 \mathrm{~min}$ to synthesize the second-strand cDNA. All libraries were sequenced by Illumina Hiseq X Ten (Illumina, Inc.).

Bioinformatics analysis. Quality control of raw reads was evaluated by Fast-QC (http://www.bioinformatics.babraham. ac.uk/projects/fastqc/). The remaining clean reads were mapped to the reference human genome (GRCh38) using HISAT2 software (version 2.1.0; https://daehwankimlab.github. io/hisat2/) and to predict the circRNAs, ACFS2 (https://github. com/arthuryxt/acfs) was used to identify and quantify circRNA from these mapping splice junction reads. The differentially 
Table I. Primer sequences for circSCYL2 in breast cancer cells.

\begin{tabular}{llcc}
\hline Primer & \multicolumn{1}{c}{ Sequences, 5'-3' $^{\prime}$} & Product expected length $/ \mathrm{bp}$ & $\mathrm{Tm} /{ }^{\circ} \mathrm{C}$ \\
\hline Divergent primer-F & CCTTCCCCTATATCTCCAGACATTA & 184 & 60 \\
Divergent primer $-\mathrm{R}$ & GACAGGATTTCCCATTACAGCA & & 60 \\
Convergent primer-F & CTTCTTACTGTCCAGCATCCTTTAG & 127 & 60 \\
Convergent primer-R & CTGGAGATATAGGGGAAGGTAGATT & & 60 \\
\hline
\end{tabular}

circ, circular; Tm, melting temperature; R, reverse; F, forward; bp, base pair.

expressed circRNAs between EMT and blank were analyzed using the DE-Seq algorithm, and significant differences were defined as absolute $\mid \log 2$ fold-changel $>1$, with a false discovery rate $(\mathrm{FDR})<0.05$. The miRanda/RNAhybird was used to predict the relationship between all miRNAs and differential mRNA and differential circRNA (the intersection of the two pieces of prediction software) was used as the final target gene prediction result. The parameters set in miRanda were energy miranda $<-20$ and score miranda $>150$, and in RNAhybird was energy RNAhybird <-25. Function and pathway analysis of these target genes of differentially expressed circRNAs were assessed using Gene Ontology (GO) (http://www.geneontology.org) and the Kyoto Encyclopedia of Genes and Genomes (KEGG) (http://www.genome.jp/kegg) databases, respectively. The significance level was assessed using the Fisher test and the significant enrichment was obtained by considering a $\mathrm{P}<0.05$. circRNA/miRNA/mRNA interactions were subsequently investigated and the circRNA-miRNA-mRNA network was constructed by Cytoscape software (version 3.6.1; https://cytoscape.org).

$R T-q P C R$. Total RNA was extracted and quantified and then reverse transcribed into complementary DNA (cDNA) using a Reverse Transcription kit (K1622; Thermo Fisher Scientific, Inc.). The procedure of reverse transcription was $25^{\circ} \mathrm{C}$ for $5 \mathrm{~min}, 42^{\circ} \mathrm{C}$ for $60 \mathrm{~min}, 70^{\circ} \mathrm{C}$ for $5 \mathrm{~min}$. Primers (Table SI) for actin, E-cadherin, Vimentin, GAPDH, circRNAs were obtained from Shanghai Sangon Pharmaceutical Co., Ltd., and the RT-qPCR analysis was performed using Applied Biosystems; Thermo Fisher Scientific, Inc. (ABI Q6). The kit used for qPCR was 2X SYBR Green PCR Master Mix (Roche Diagnostics) and the thermocycling conditions were $95^{\circ} \mathrm{C}$ for $10 \mathrm{~min}, 45$ cycles of $95^{\circ} \mathrm{C}$ for $15 \mathrm{sec} ; 60^{\circ} \mathrm{C}$ for $60 \mathrm{sec}$. The temperature of melt curve was 60 to $99^{\circ} \mathrm{C}$. The actin and GAPDH was used as an internal control. Relative quantification and calculation were done through the comparative threshold (Cq) cycle method $\left(2^{-\Delta \Delta \mathrm{Cq}}\right)(26)$.

PCR and Sanger sequencing. cDNA extraction was performed as previously described, gDNA was extracted using Genomic DNA Isolation kit (Bio-Rad Laboratories, Inc.). Divergent primers and convergent primers listed in Table I were designed for the PCR reaction. The cDNA and gDNA PCR products were observed using 2\% agarose gel electrophoresis. CircRNAs of purified PCR products were validated by Sanger sequencing and the results were compared with the reference sequence (UCSC chr12_100298175_100282943_+15232-SCYL2).
Human breast cancer and normal tissues. A total of 20 paired breast cancer specimens and normal adjacent tissues $(>2 \mathrm{~cm}$ from the edge of tumor tissue) were obtained from patients undergoing surgical resection at the Second Affiliated Hospital of Nanchang University, between January 2018 and July 2019). The age range of patients is 40 to 65 years old, with a median age of $51 \pm 2.42$ years old. The patients had the inclusion criteria as follows: i) Diagnosed as breast cancer by pathological confirmation; ii) without radiotherapy or chemotherapy before surgery; iii) complete clinical and pathological data. The exclusion criteria were as follows: i) Combined with other tumors and serious diseases and ii) incomplete clinical and pathological data. All the patients provided written consent and the study was approved by the Ethics Committee of the Second Affiliated Hospital of Nanchang University. Tissue fragments were immediately frozen in liquid nitrogen at the time of surgery and stored at $-80^{\circ} \mathrm{C}$.

Plasmids construction and cell transfection. Human circSCYL2 cDNA with BamHI (GGATCC) and EcoRI (GAATTC) restriction sites was synthesized by Yingbio Technology and cloned into the pcDNA circRNA vector, which contained a front circular frame and a back circular frame. MCF-7 and MDA-MB-231 cells were seeded in 6-well plates at a concentration of $3 \times 10^{5}$ cells/well; when the cells grew to $80-90 \%$ confluency, the fresh culture medium was exchanged from each well. Transfection of $2 \mathrm{ug}$ vector was carried out using Lipofectamine 2000 (Invitrogen; Thermo Fisher Scientific, Inc.) according to the manufacturer's protocol. An empty vector was used as the negative control. After transfection for $48 \mathrm{~h}, \mathrm{RT}-\mathrm{qPCR}$ was performed to detect the expression level of circSCYL2.

Transwell assay. Transwell migration was performed using $0.8 \mu \mathrm{m}$ 24-well transwell chambers (353097; FALCON ${ }^{\mathrm{TM}}$, Corning, Inc.) and transwell invasion was performed using BioCoat $^{\mathrm{TM}}$ Matrigel $^{\circledR} 0.8 \mu \mathrm{m}$ 24-well trans-well chamber (354480, BioCoat; Thermo Fisher Scientific, Inc.). After transfection for $48 \mathrm{~h}$, the cells were digested, centrifuged with $200 \mathrm{x} \mathrm{g}$ at room temperature for $3 \mathrm{~min}$ and resuspended in a serum-free medium to adjust the concentration to $1.5 \times 10^{5}$ cells $/ \mathrm{ml}$. A cell suspension of $500 \mu \mathrm{l}$ was inoculated into the upper chamber and $700 \mu 1$ medium containing 10\% FBS was added to the lower chamber as a chemoattractant. After incubation for $24 \mathrm{~h}$, the transwell chamber was clipped and the invaded or migrated cells were fixed with formaldehyde at room temperature for $30 \mathrm{~min}$ and stained using crystal violet at 
A

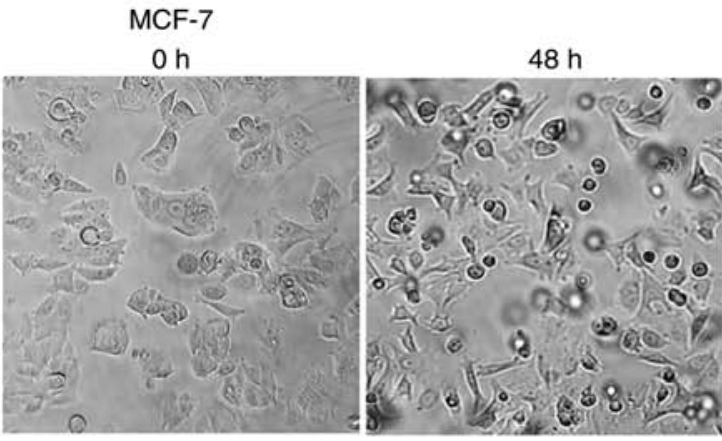

MDA-MB-231

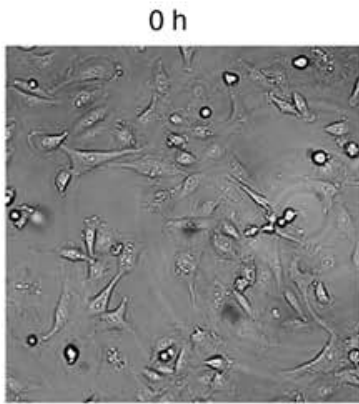

$48 \mathrm{~h}$

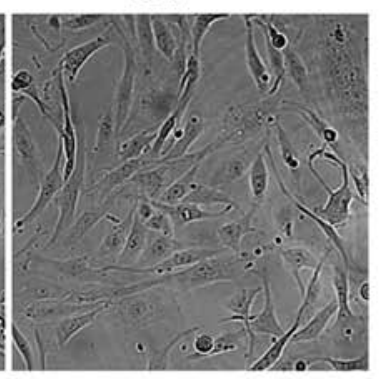

B
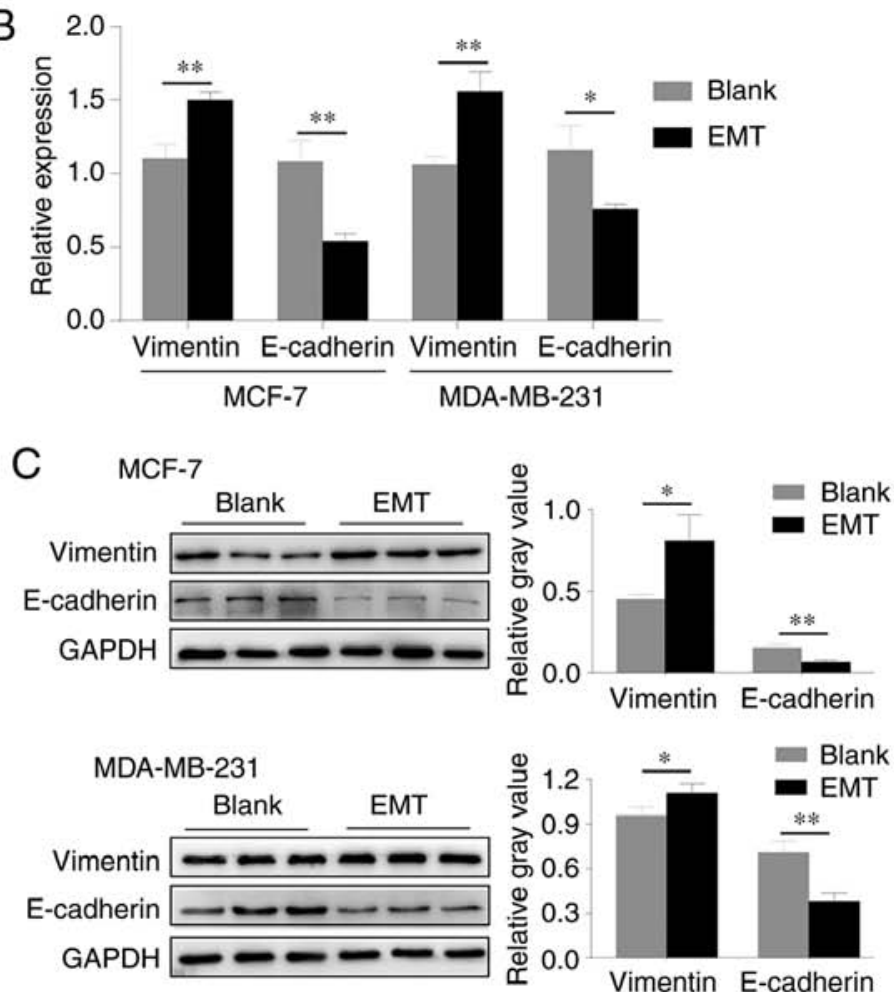

Figure 1. Establishment of EMT model in breast cancer cells. (A) Representative micrographs (magnification, x100) of tumor cells after 0 and $48 \mathrm{~h}$ transforming growth factor- $\beta 1$ induction. (B) Reverse transcription-quantitative PCR validated the expression levels of E-cadherin and Vimentin in EMT and blank groups of two cells. Experiments were performed in quadruplicate. The value of $2^{-\Delta \Delta C q}$ was used to show the expressional level of RNAs. Data are expressed as mean \pm SD. (C) Protein levels of E-cadherin and Vimentin were measured by western blot analysis. GAPDH was used as an internal control. ${ }^{*} \mathrm{P}<0.05$ and ${ }^{* *} \mathrm{P}<0.01$. EMT, epithelial mesenchymal transition; circ, circular; E, epithelial.

room temperature for $30 \mathrm{~min}$. The cells on the upper surface of the membrane were wiped off with cotton swabs and those on the lower surface were photographed and counted by ImageJ software (version 1.8.0). The numbers of invaded and migrated cells were counted in 3 randomly selected fields.

Statistical analysis. RT-qPCR was repeated at least in three independent experiments in every sample. Data were presented as mean $\pm \mathrm{SD}$ of three or more independent experiments. The significant expression of circSCYL2 in breast cancer tissues and normal tissues was valued by paired Student's t-test, and other significance was assessed with unpaired Student's t-test using SPSS 24.0 software (IBM, Corps.). $\mathrm{P}<0.05$ was considered to indicate a statistically significant difference.

\section{Results}

Establishment of EMT model in breast cancer cells. After TGF- $\beta 1$ stimulation, breast cancer cells gradually displayed the characteristics of mesenchymal cells; the cells became elongated and circular or polygonal progressively with the extension of pseudopodia, and they became spindle-shaped, while their intercellular space grew wider, and the cells loosened up with an irregular distribution (Fig. 1A). To examine the establishment of EMT, the expression of E-cadherin and Vimentin in TGF- $\beta 1$ stimulated cells was detected by RT-qPCR and western blotting analysis. It was shown that the mRNA and protein expression levels of E-cadherin significantly decreased, whereas the expression of Vimentin was statisti- cally elevated when compared to the blank (Fig. 1B and C). These results suggest that EMT of breast cancer cells has been established successfully by TGF- $\beta 1$ induction.

Analysis of circRNA transcript data and differentially expressed circRNAs. To investigate the roles of circRNA in EMT of the breast cancer induced by TGF- $\beta$, the present study analyzed the circRNA expression profiles of MCF-7 and MDA-MB-231 cells using RNA sequencing (RNA-seq) analysis. The sequencing experimental flow chart of this study is shown in Fig. 2A. A total of $\sim 77$ (MCF-7-blank), 105 (MCF-7-EMT), 90 (MDA-MB-231-blank) and 95 (MDA-MB-231-EMT) million clean reads were obtained. A total of $87-97 \%$ of these reads were mapped to the reference genome and $\sim 18-22 \%$ of clean reads were realigned to determine junction reads. A total of 23,347 circRNAs were predicted from junction reads, among these, 10,715 and 10,412 circRNAs were detected in the blank and EMT group, respectively in MCF-7 cells, while in MDA-MB-231 cells, the data was 5,035 and 5,405, respectively (Table II).

The Venn diagram in Fig. 2B reveals circRNAs distribution and helps understanding the shared and unique circRNAs between blank and EMT. In the current study 7,057 and 6,754 circRNAs were detected exclusively in blank and EMT of MCF-7 cells, respectively, and only 3,658 circRNAs were shared between the two groups. Also, 3,717 and 4,087 circRNAs were exclusively expressed in blank and EMT of MDA-MB-231 cells respectively, only 1,318 circRNAs overlapped in both groups (Fig. 2B). These circRNAs of the blank 

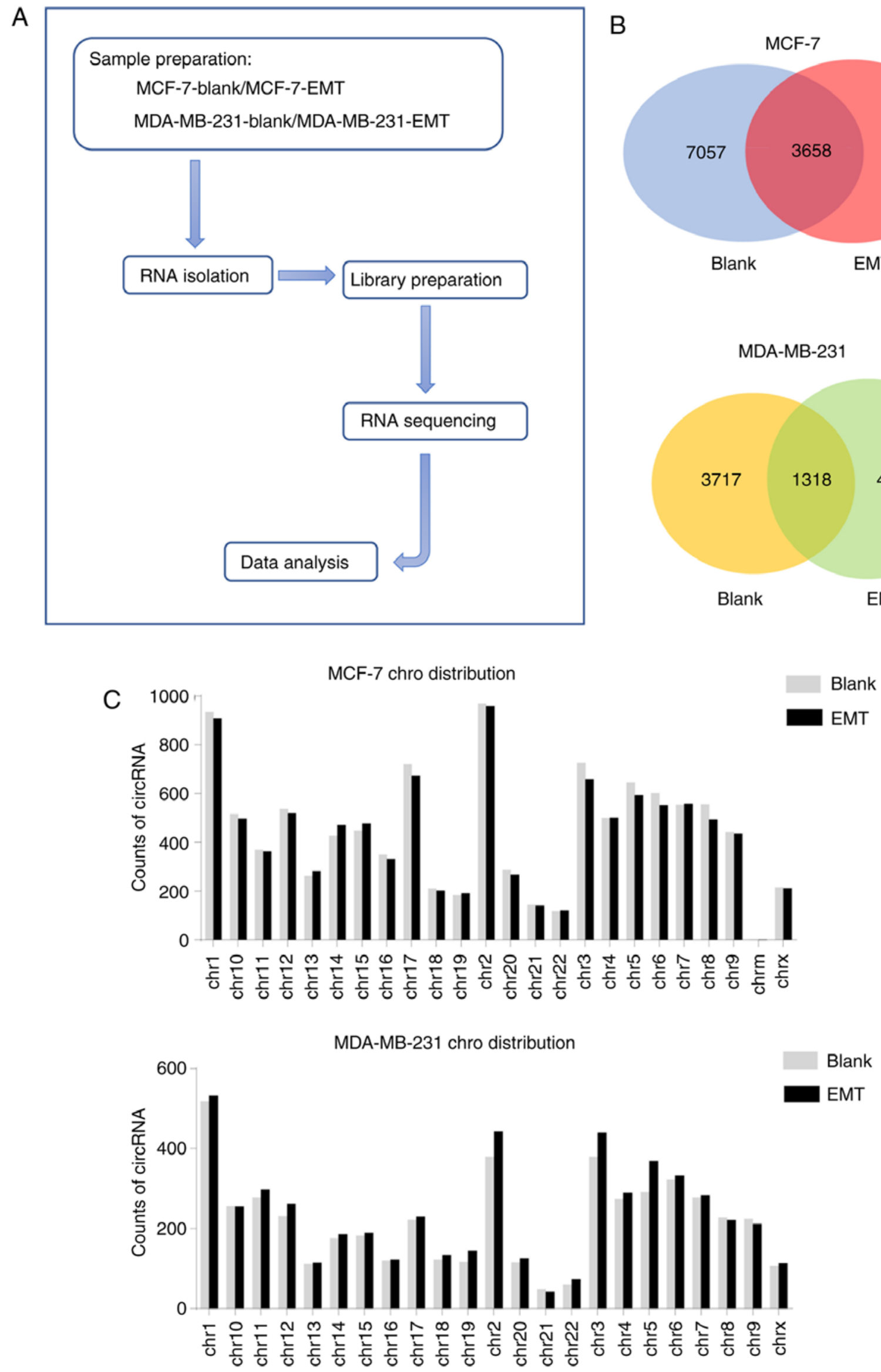

Figure 2. Analysis of circRNA transcript data and differentially expressed circRNAs. (A) The flowchart of RNA-sequencing. (B) Venn diagram shows circRNAs distributions between blank and EMT of two cells. The blue and red circles represent the number of circRNAs in MCF-7 blank and EMT cells, respectively. The yellow and green circles represent the number of circRNAs in MDA-MB-231 blank and EMT cells, respectively. (C) Chromosomal location of circRNAs between blank and EMT lines of MCF-7 and MDA-MB-231 individually. 

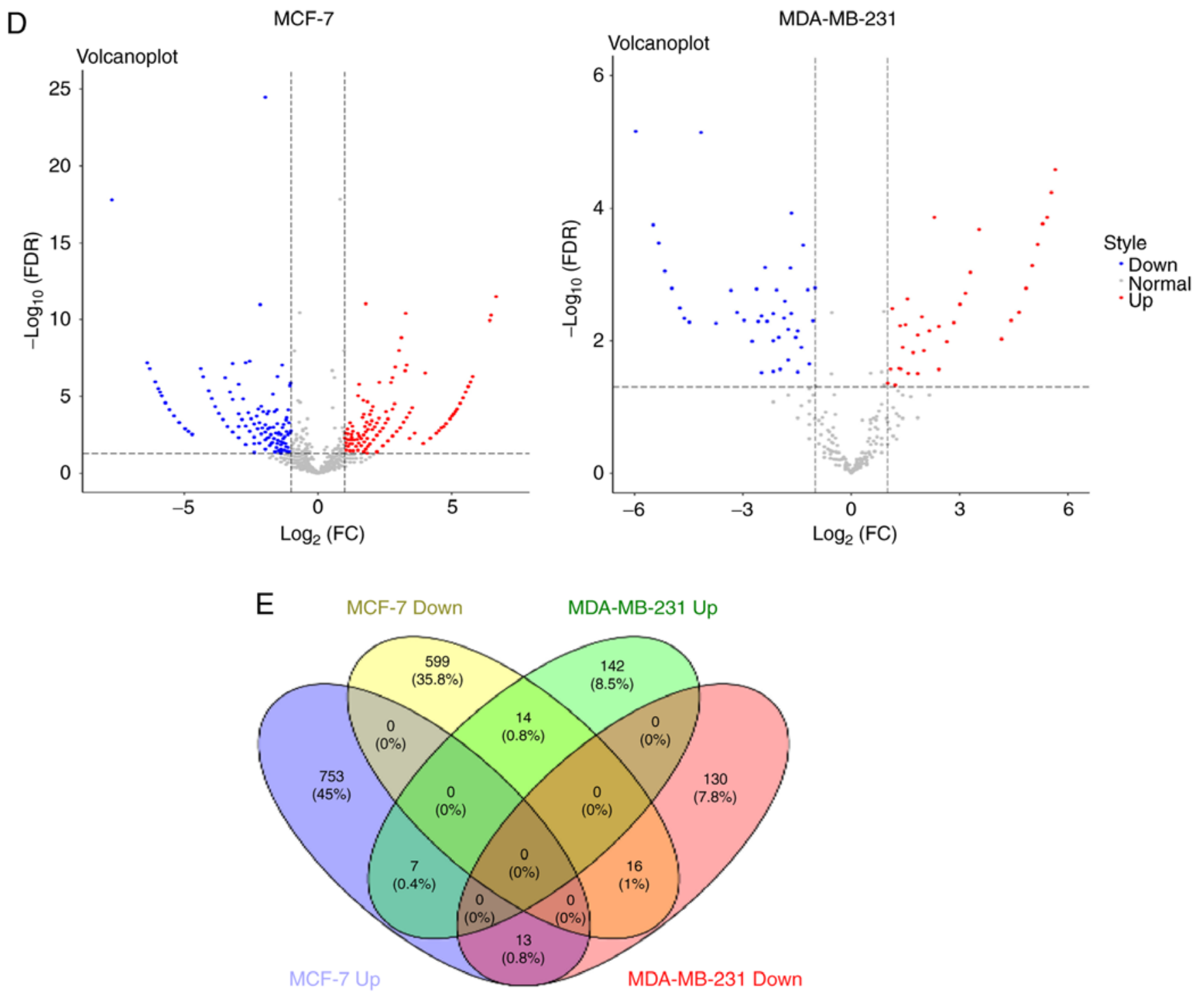

Figure 2. Continued. (D) Volcano plot visualizing the differential expression of circRNAs between blank and EMT group. X-axis: Log 2 (fold-change) of circRNA expression levels between EMT and blank groups of two breast cells. Y-axis: The FDR value $\left(-\log _{10}\right.$ transformed) of circRNAs. The blue dot indicates $>2$-fold decreased and red dot indicates $>2$-fold increased expression of the dysregulated circRNAs. (FDR $<0.05$ ). (E) Venn diagram comparison of significantly dysregulated circRNAs in two tumor cells. The purple and yellow circles represent up- and downregulated circRNAs respectively in MCF-7 EMT cells. The green and red circle represent up- and downregulated circRNAs, respectively in MDA-MB-231 EMT cells. EMT, epithelial mesenchymal transition; circ, circular; FDR, false discovery rate.

Table II. Summary of the sequencing reads alignment to the reference genome.

\begin{tabular}{lcccc}
\hline Type & MCF-7-blank & MCF-7-EMT & MDA-MB-231-blank & MDA-MB-231-EMT \\
\hline All reads & 77697606 & 105327346 & 90555230 & 95327320 \\
Mapped reads & $75372155(97 \%)$ & $97792240(93 \%)$ & $79454343(88 \%)$ & $83504290(87 \%)$ \\
Junction Mapped reads & $15980915(21 \%)$ & $23008054(22 \%)$ & $16930465(18 \%)$ & $18926756(20 \%)$ \\
cirRNA number reads & 10,715 & 10,412 & 5,035 & 5,405 \\
\hline
\end{tabular}

circ, circular; EMT, epithelial mesenchymal transition.

and EMT in two cell lines were widely distributed among all chromosomes; the number of circRNAs in each chromosome showed no significant difference between the blank and EMT groups (Fig. 2C).
Differentially expressed circRNAs were identified between blank and EMT of the two cells to analyze the role of circRNA in EMT. The Volcano plot exhibited circRNAs profiles that were significantly differentially expressed between the blank 


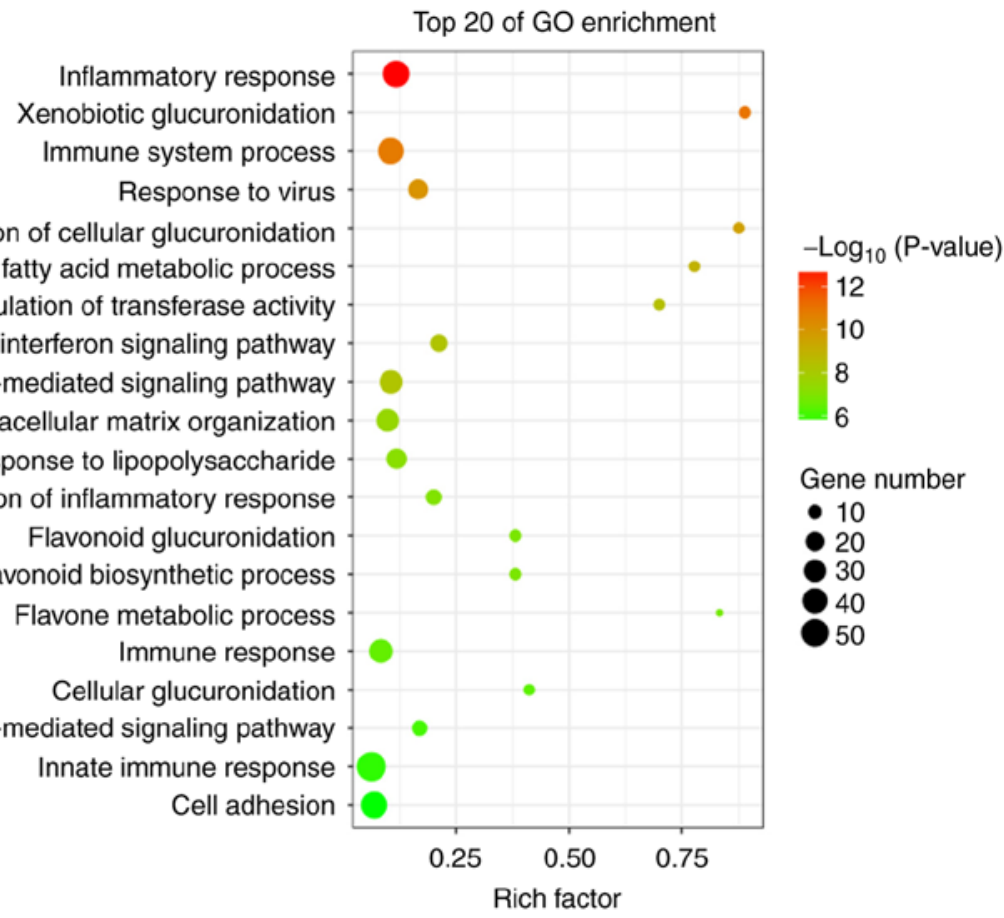

MDA-MB-231

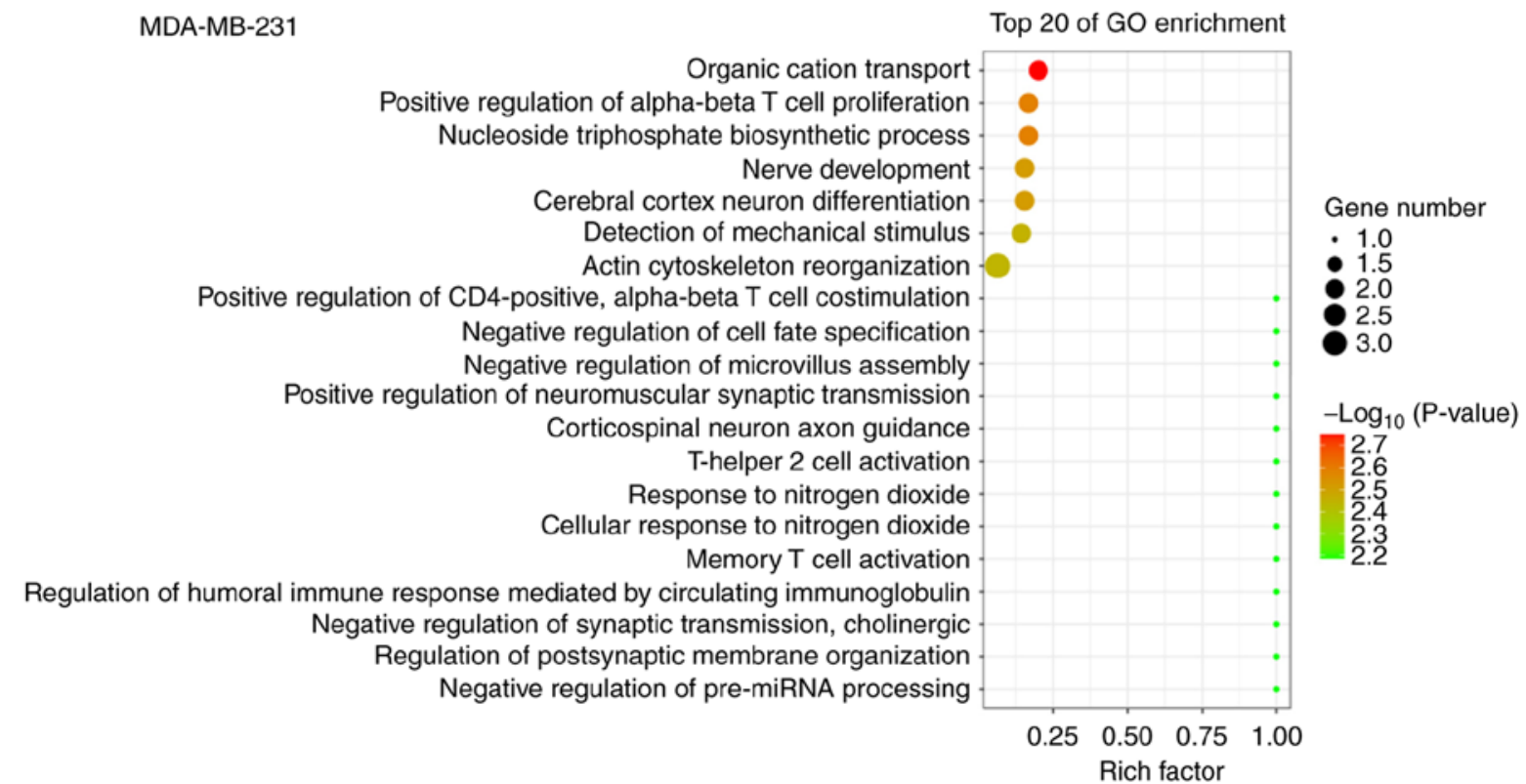

Figure 3. Scatterplot of top 20 distinguishable enriched GO and KEGG terms of differentially expressed circRNAs. (A) The top 20 enriched GO terms of the differentially expressed circRNAs. The Y-axis represents GO name and the X-axis represents the Rich factor. The size of each bubble represents the number of differentially expressed genes enriched in GO terms, and color represents - $\log 10$ (P-value).

and EMT groups of two cells. There were 1,402 significantly differentially expression circRNAs between EMT and blank of MCF-7 cells, among them, 773 circRNAs were significantly upregulated and 629 were downregulated in the EMT group. While in MDA-MB-231 cells, 322 circRNAs were significantly differentially expressed between the blank and EMT groups, of which, 163 circRNAs were upregulated and 159 were downregulated within blank and EMT (Fig. 2D). The Venn diagram shows the common and unique dysregulated circRNAs in the two cells. Among all these significantly dysregulated circRNAs, only 7 (0.4\%) circRNAs were significantly upregulated and $16(1 \%)$ circRNAs were significantly downregulated in both the MCF-7 EMT group and the
MDA-MB-231 EMT group, compared with the corresponding blank groups (Fig. 2E).

GO and KEGG analysis of differently expressed circRNAs. To explore the potential functional roles of circRNAs, a circRNA-mediated competing endogenous RNA network in tumor cells was constructed to predict differentially expressed mRNAs that were targeted by the differentially expressed circRNAs. GO analysis was applied to explore the biological functions of hostmRNA. Interestingly, it was found that the most significantly enriched functional terms were associated with the immune/inflammatory response and cell adhesion/adherence junctions in both MCF-7 and MDA-MB-231 cells, such 
B $\quad$ MCF-7

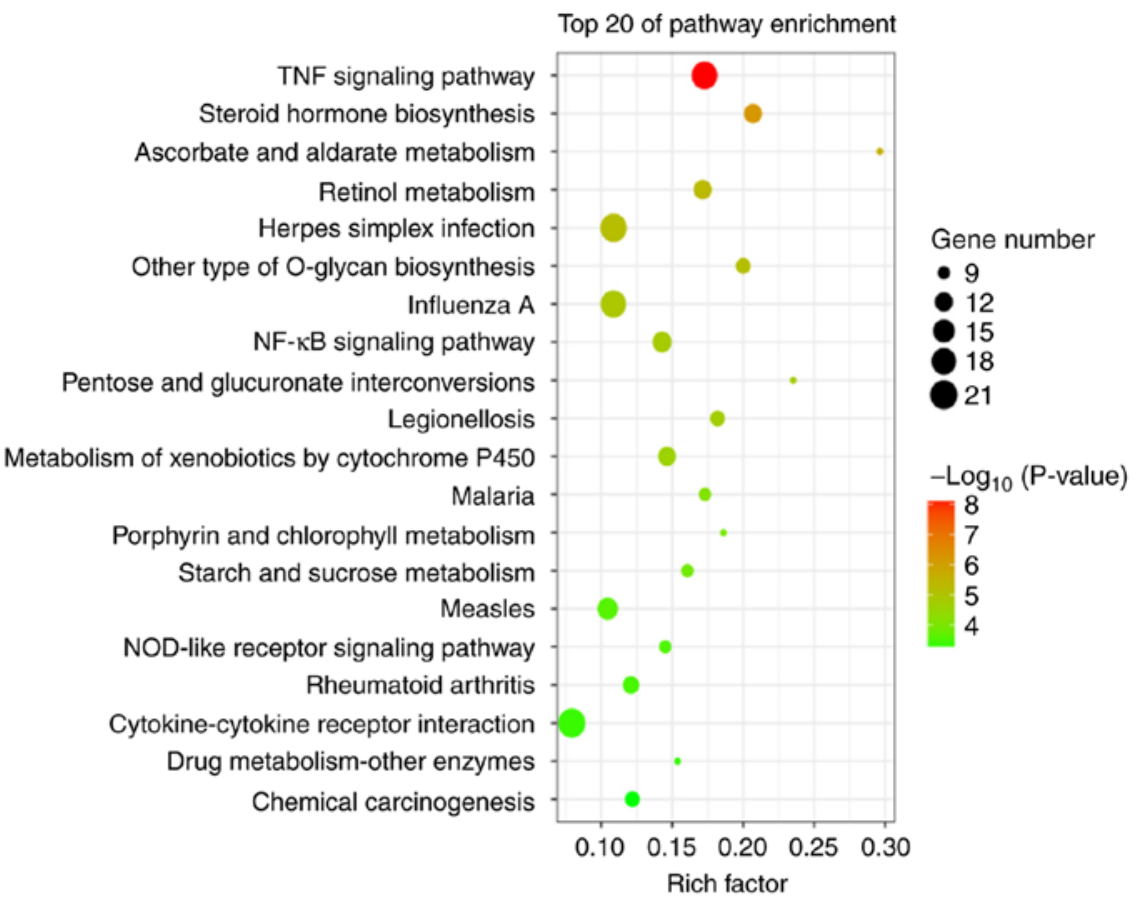

MDA-MB-231

Top 20 of pathway enrichment

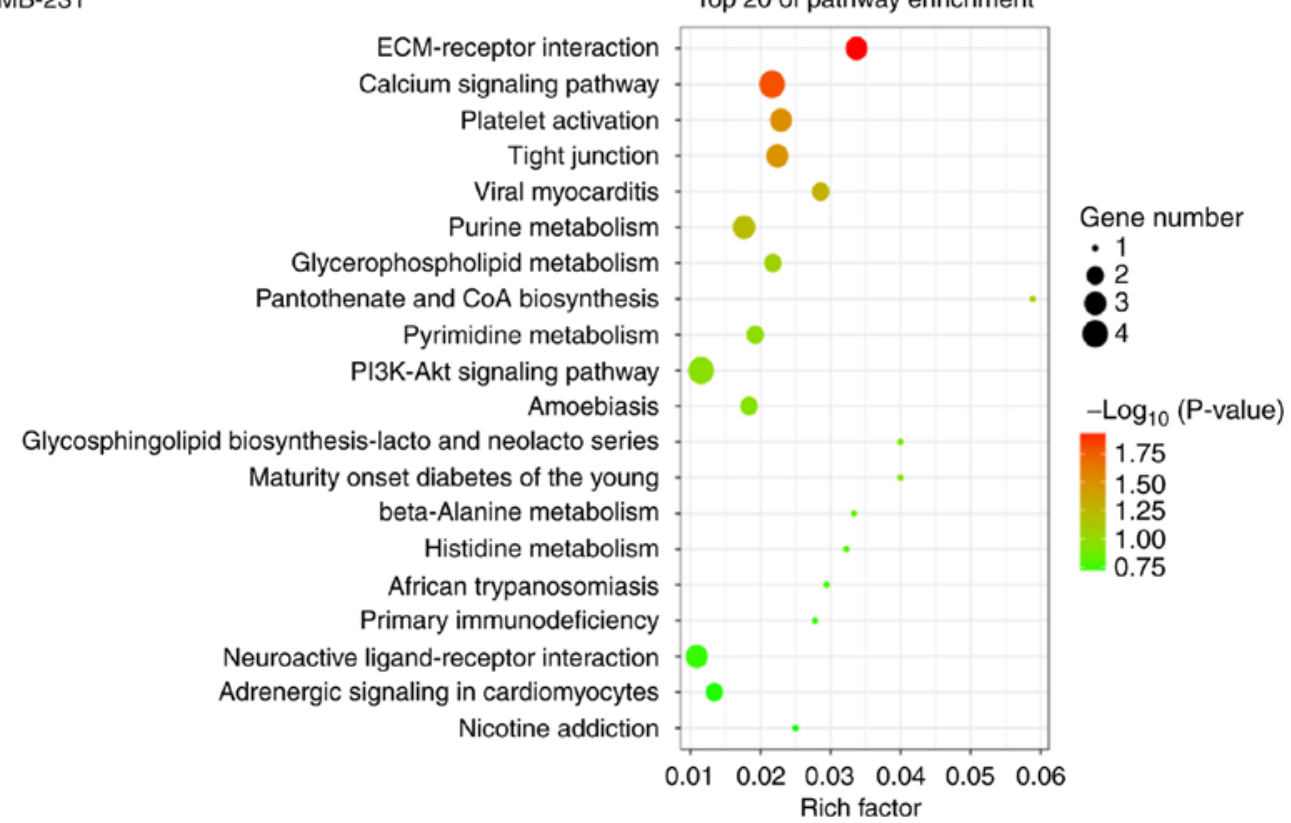

Figure 3. Continued. (B) The top 20 enriched KEGG pathways of the differentially expressed circRNAs. The Y-axis represents pathway name and the X-axis represents the Rich factor. The size and color of each bubble represents the number of differentially expressed genes enriched in the pathway and -log 10 (P-value), respectively. circ, circular; GO, gene ontology; KEGG, Kyoto Encyclopedia of Genes and Genomes.

as 'inflammatory response', 'immune system process', 'cell adhesion', 'positive regulation of $\alpha-\beta$ T cell proliferation', and 'actin cytoskeleton reorganization' (Fig. 3A).

The host genes were classified by KEGG function annotations to analyze the pathways (Fig. 3B). In MCF-7 cells, KEGG analysis consistently revealed various signaling pathways involved in regulating tumor growth, such as 'TNF signaling pathway', 'Steroid hormone biosynthesis', 'NF- $\mathrm{B}$ signaling pathway' and 'PI3K-AKT pathway'. Similarly in MDA-MB-231 cells, pathways such as 'ECM-receptor interaction' and 'Calcium signaling pathway' were enriched, which were reported to be associated with tumor cell proliferation and migration (27-31).
Construction of the circRNA-miRNA-mRNA interaction network. Next, to appraise the valuable circRNAs, the expression changes of the simultaneously dysregulated circRNAs in EMT and blank groups of two cells are shown in Fig. 4A. Although differentially expressed circRNAs have been proposed to be closely associated with EMT $(12,15)$, the roles of circRNAs in breast cancer EMT remain unclear. The present study aimed to identify novel circRNAs that regulate EMT of breast cancer cells. A total of five circRNAs were screened from these simultaneously dysregulated circRNAs as the following criteria: i) Up or downregulation was identified in EMT groups of the two cells, compared with their 
A

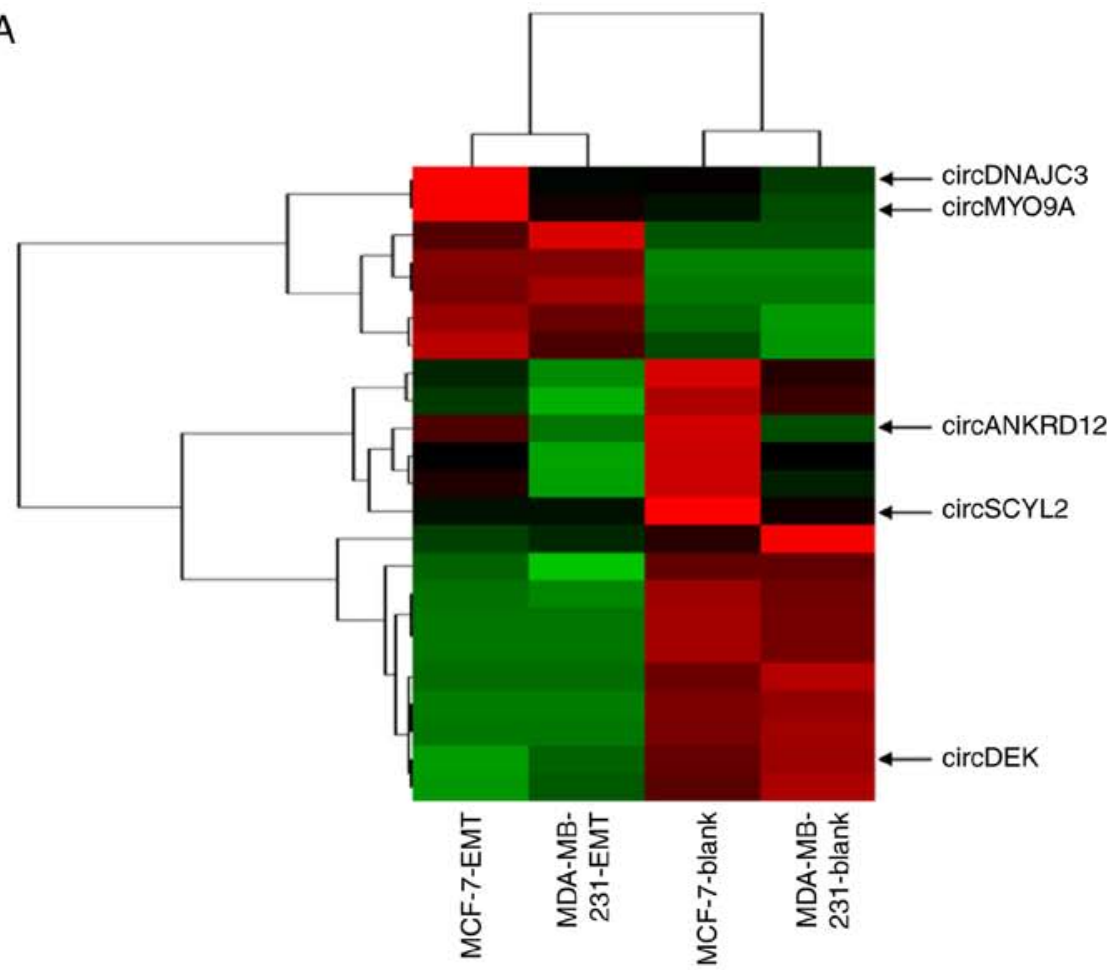

B

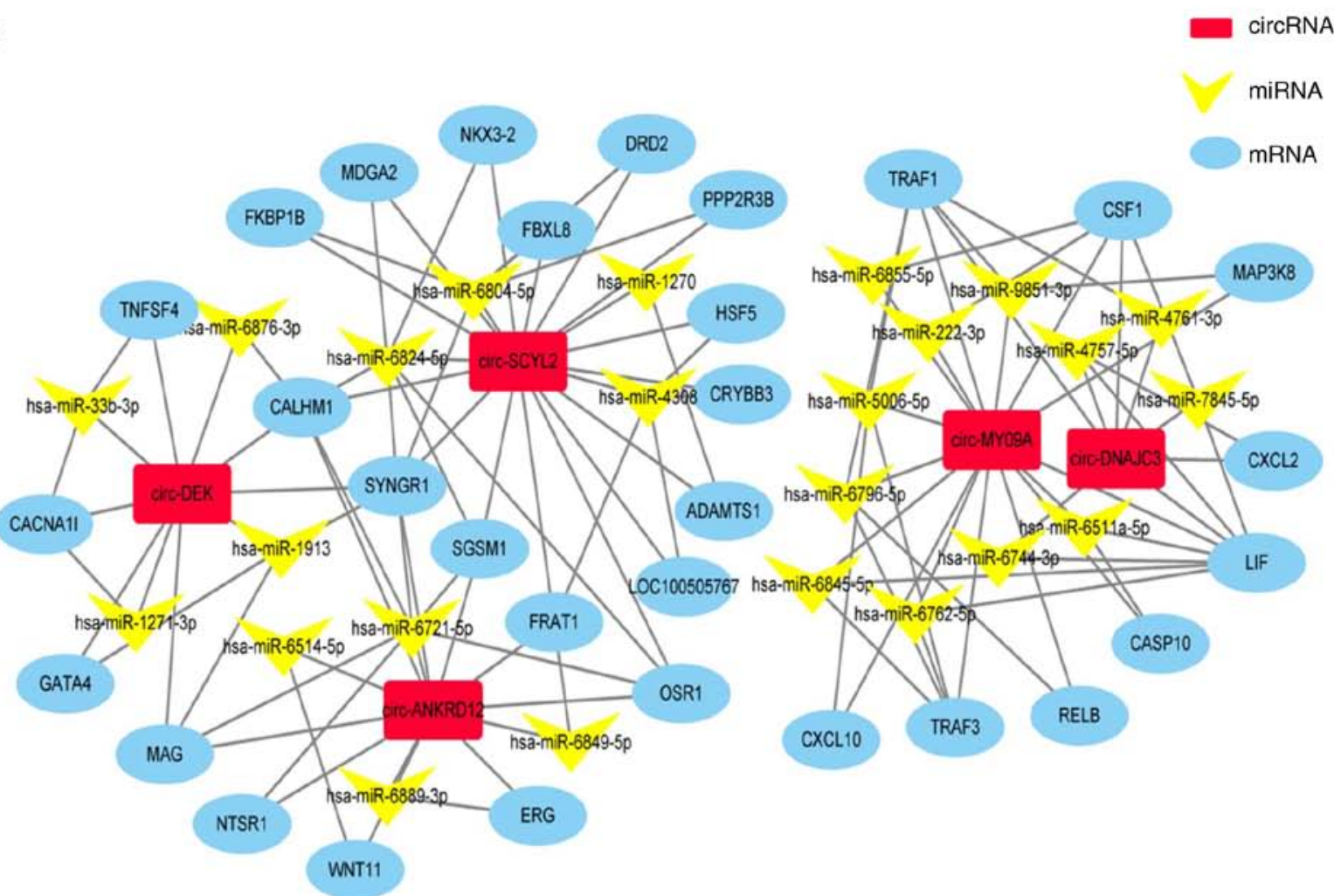

Figure 4. Construction of the circRNA-miRNA-mRNA interaction network. (A) Hierarchical cluster of the simultaneously dysregulated circRNAs expression profiles in the EMT and blank groups of two cells. The heatmap colors represent relative circRNA abundance in each sample. The color scale runs from green (low intensity) to red (strong intensity). (B) The circRNA-miRNA-mRNA network for the 5 circRNAs. chr15 72046634 72007826 -38808MYO9A (circMY09A), chr13_95763953_95757644_+6309-DNAJC3 (circDNAJC3), chr18_9221999_9208657_+13342-ANKRD12 (circANKRD12), chr 6_18258405_18236452_-21953-DEK (circDEK), chr12_100298175_100282943_+15232-SCYL2 (circSCYL2). circ, circular; miRNA, microRNA; EMT, epithelial-mesenchymal transition.

blank groups, respectively; ii) higher fold-change and iii) more abundant expression level. Heatmaps assessed and compared the expression changes of circRNAs in EMT and blank groups of two cells (Fig. 4A). The circRNA-miRNA-mRNA network was constructed by predicting the differentially expressed genes of five circRNAs and some target genes of these five circRNAs, such as OSR1, DRD2, MAP3K8, and TNFSF4 that are related to cancer (Fig. 4B) (32-35). 

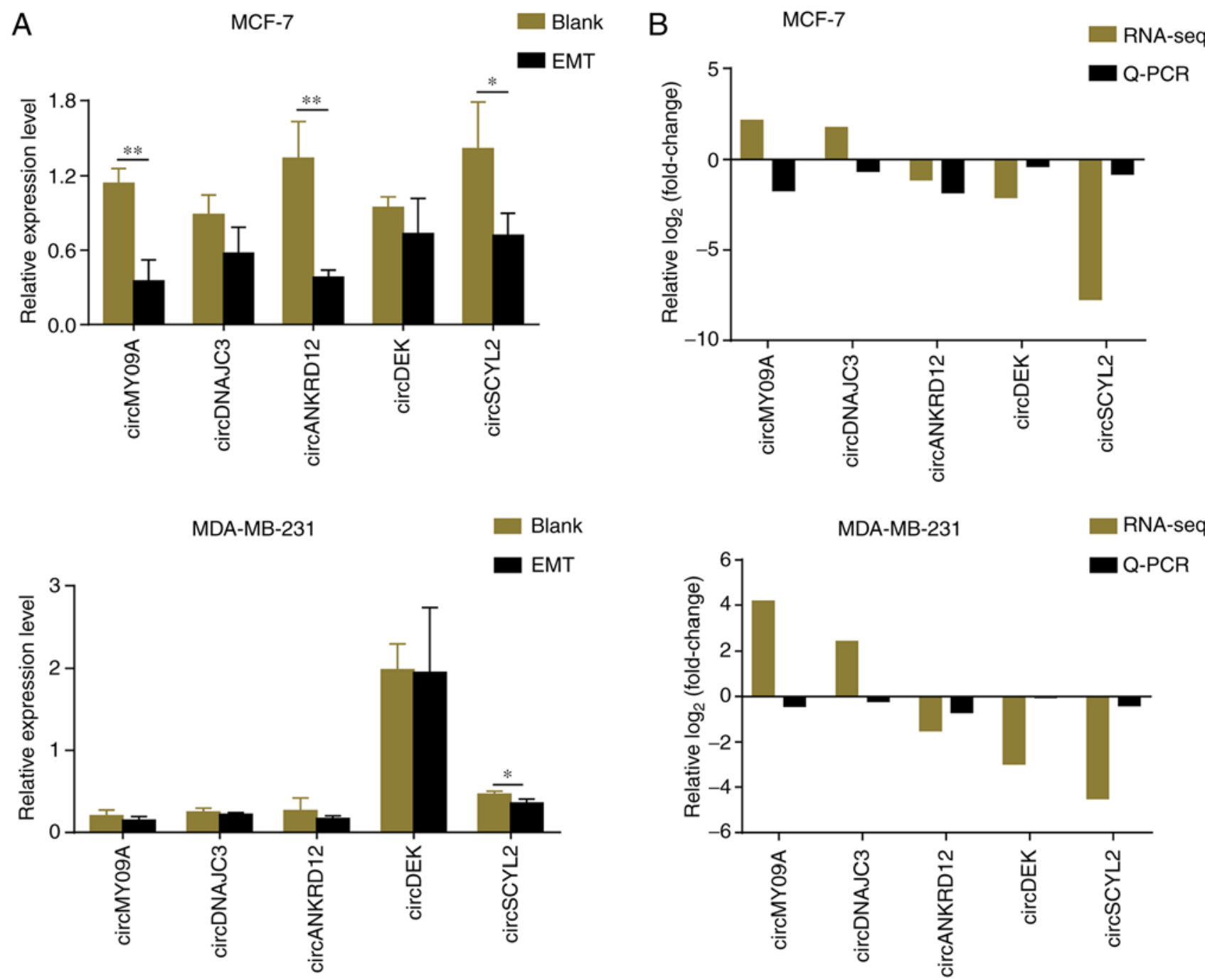

Figure 5. Verification of candidate circRNAs by RT-qPCR. (A) The relative expression levels of the selected significantly differentially expressed circRNAs were validated by RT-qPCR. The value of $2^{-\Delta \Delta C q}$ was used to show the expression level of circRNAs. Data are expressed as mean \pm SD. ${ }^{*} \mathrm{P}<0.05$ and ${ }^{* *} \mathrm{P}<0.01$ (B) Relative $\log _{2}$ (fold-change) of circRNAs expression between RNA-sequencing and RT-qPCR results. GAPDH was used as the internal control. RT-q, reverse transcription-quantitative; circ, circular; EMT, epithelial-mesenchymal transition.

Verification of candidate circRNAs by RT-qPCR. The sequencing data of five candidate circRNAs was validated using RT-qPCR in EMT groups of MCF-7 and MDA-MB-231 cells and matched with their blanks, respectively (Fig. 5A). On comparing RNA-seq results with RT-qPCR information, it was found that three of the five circRNAs were downregulated in EMT groups, which was consistent with RNA sequencing. As the stability of circRNA sequencing is not the same as mRNA sequencing, the results of two circRNAs between qPCR and RNA-seq were not matched. Notably, circSCYL2 was the most downregulated circRNA (Fig. 5B).

circSCYL2 is significantly downregulated in breast cancer. Due to the significant downregulation of circSCYL2 in EMT groups compared with that in the blank group, the current study next assessed the exon structure of circSCYL2. Convergent primers were designed to amplify SCYL2 mRNA and divergent primers to amplify circSCYL2. Using cDNA and genomic DNA (gDNA) from breast cancer cell lines as templates, circSCYL2 was only amplified by divergent primers in cDNA, and no amplification product was observed in gDNA (Fig. 6A). circSCYL2 derived from exon 2 to 4 of the SCYL2 gene with a length of $508 \mathrm{nt}$, which was confirmed the back-spliced junction in the RT-qPCR product of circSCYL2 with the expected size by Sanger sequencing (Fig. 6B and C). To investigate the expression of circSCYL2 in breast cancer, the expression level of circSCYL2 in 20 pairs of human breast cancer tissues and normal breast tissues were detected, and the results demonstrated that the expression of circSCYL2 was significantly decreased in breast cancer tissues compared with in normal breast tissues (Fig. 6D).

Overexpression of circSCYL2 inhibits the migration and invasion of breast cancer cells. The results of the present study demonstrated that circSCYL2 is downregulated in breast cancer tissues and cell lines. To investigate the function of circSCYL2 in breast cancer progression, the circSCYL2 expression construct was transfected into MCF-7 and MDA-MB-231 cells, and the expression of circSCYL2 was significantly increased. Meanwhile, the expression of E-cadherin mRNA was enhanced in MCF-7 and MDA-MB-231 cells with over-expression of circSCYL2, while the expres- 


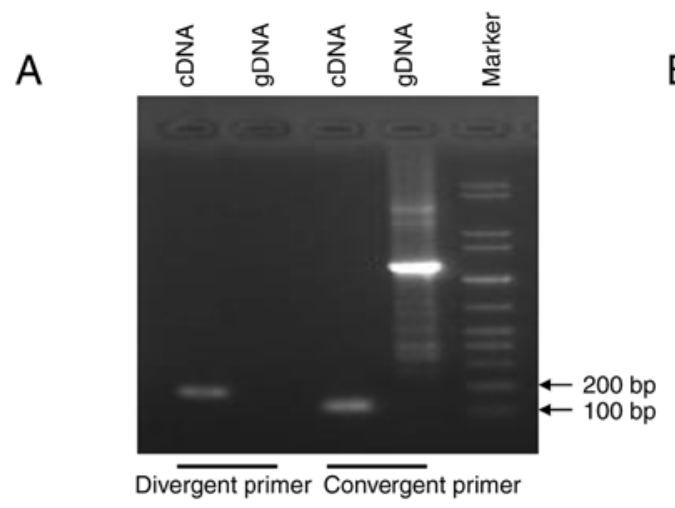

B
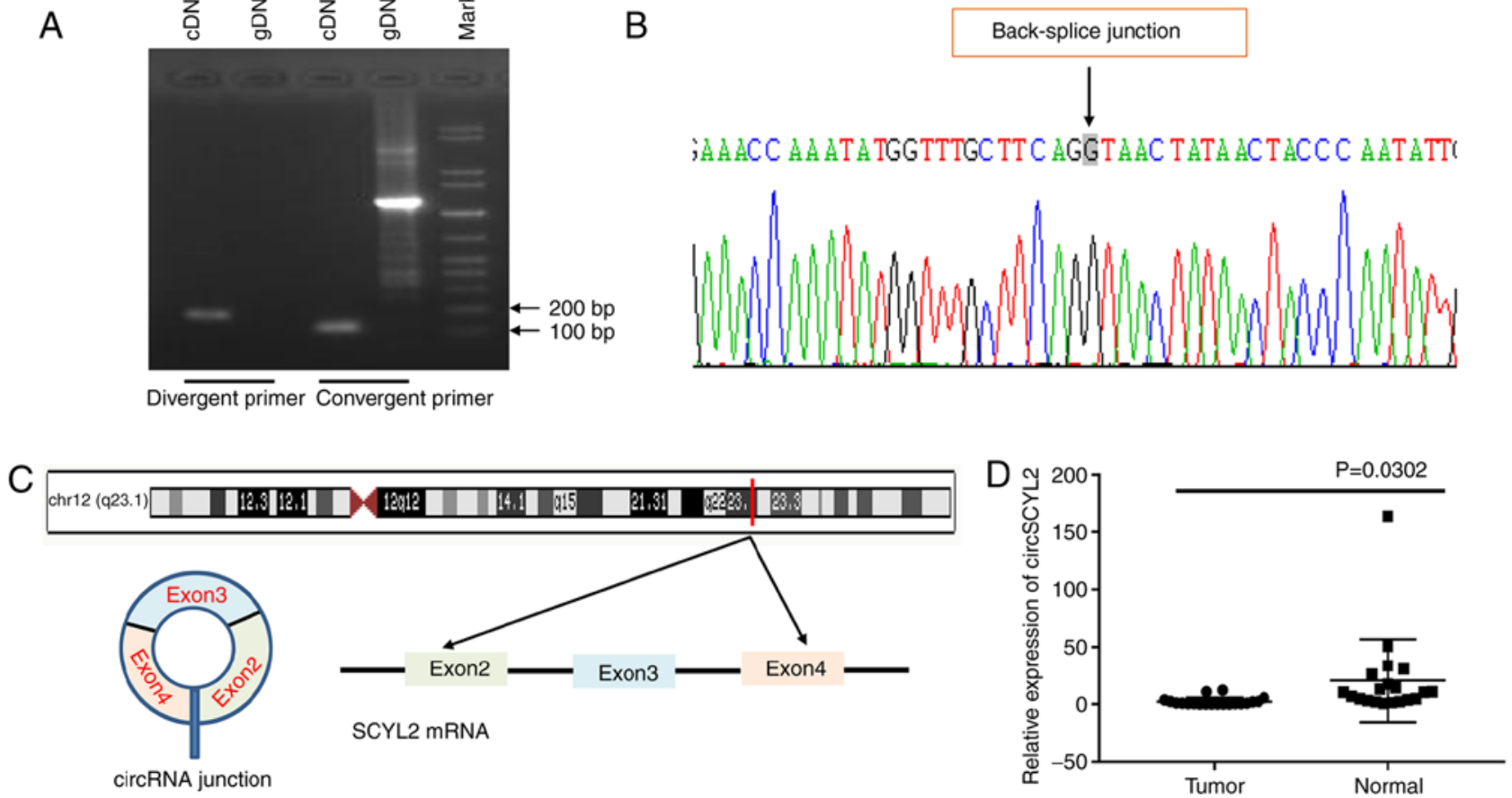

Figure 6. circSCYL2 is significantly downregulated in breast cancer. (A) PCR analysis with divergent primers and convergent primers for circSCYL2 and its linear isoform SCYL2 in cDNA and gDNA. Convergent primers were used to amplify mRNA. gDNA was used as control. (B) RT-qPCR products were used to verify the existence of circSCYL2 and its back-splice junction by Sanger sequencing. (C) Schematic illustration showing the circularization of SCYL2 (NM_001317784.1) exon 2-4 form circSCYL2. (D) RT-qPCR assay confirmed the low expression of circSCYL2 in 20 pairs of human breast cancer tissues compared with their adjacent normal tissues. The relative levels of circSCYL2 were expressed as the value of $2^{-\triangle \Delta C q}$. Data are mean \pm SD, $n=3$. GAPDH was used as control. RT-q, reverse transcription-quantitative; circ, circular; gDNA, genomic DNA.

sion of Vimentin was significantly reduced (Fig. 7A). A matrigel-coated transwell invasion assay was used to examine the abilities of cell migration and invasion. Transwell assays indicated that the migration and invasion abilities of breast cancer cell lines were also suppressed by over-expression of circSCYL2 (Fig. 7B).

\section{Discussion}

Breast cancer metastasis is the leading cause of mortality in patients; metastatic tumors can occur after a period of clinical therapy anytime from months to decades, leaving the survivors uncertain about their longer-term prognosis (36). Recent research has suggested that circRNAs are involved in various breast cancer biological procession $(37,38)$, but its function in EMT of breast cancer remains largely unclear. In this study, the whole transcriptome RNA-Seq was used to construct circRNA expression profiles in two breast cancer cells MCF-7 and MDA-MB-231 which were induced by TGF- $\beta 1$. A total of 7 upregulated circRNAs and 16 downregulated circRNAs were identified to be shared in the MCF-7-EMT and MDA-MB-231-EMT groups. Functional investigations revealed that these differently expressed circRNAs were associated with immune/inflammatory response, cell proliferation/cell adhesion functions and relative pathways. The present study identified and validated that circSCYL2 was downregulated in breast cancer tissues and cell lines; the overexpression of circSCYL2 in breast cancer cells inhibited the abilities of cell migration and invasion.

Previous studies have revealed that circRNAs play important roles in the regulation of multiple diseases, especially cancers (39-42). Aberrant expression of circRNAs was also correlated with progression, drug resistance and prognosis of cancers (21). Increasing evidence indicates that one of the important functions of circRNAs is to serve as miRNA sponges to regulate target genes, therefore the circRNA-miRNA-mRNA network was constructed to find the target genes of differentially expressed circRNAs (43). For instance, hsa_circ_0058124 promotes papillary thyroid cancer tumorigenesis and invasiveness through the NOTCH3/GATAD2A axis, elevated levels of hsa_circ_006100 in gastric cancer promote cell growth and metastasis via miR-195/GPRC5A signaling $(44,45)$. The current study identified 7 significantly upregulated circRNAs and 16 downregulated circRNAs that were shared in both the MCF-7 EMT group and the MDA-MB-231 EMT group which demonstrated that these circRNAs may play important roles in breast cancer metastasis.

circSCYL2 arose from the SCYL2 gene and consisted of the head-to-tail splicing of exon 2-4. A total of 16 differentially expressed target genes of circSCYL2 were found in the circRNA-miRNA-mRNA network and they were downregulated in MCF-7-EMT and MDA-MB-231-EMT cells. Among them, the odd-skipped related transcription factor 1 (OSR1) gene, which is located on human chromosome 2p24.1 and encodes a zinc-finger transcription factor was focused on. Previous studies suggested that OSR1 suppresses the progression of gastric cancer and renal cell carcinoma $(46,47)$. Another study also found that OSR1 was downregulated in tongue squamous cell carcinoma (TSCC) cells and specimens and that OSR1 overexpression inhibited TSCC cell migration and invasion, while its knockdown promoted TSCC cell 
A

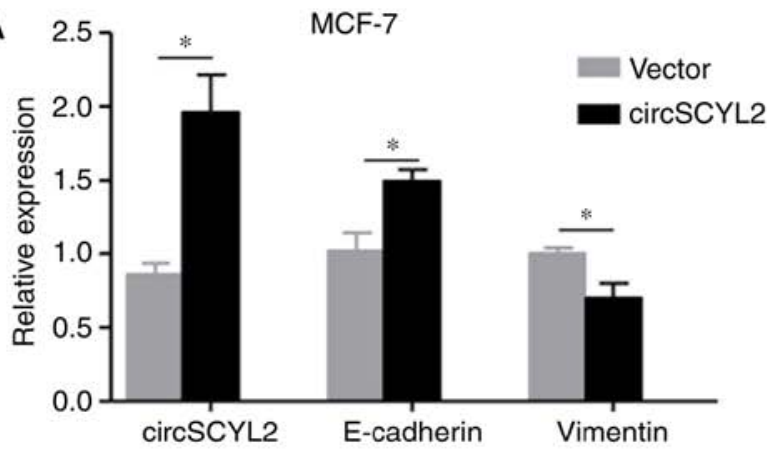

B
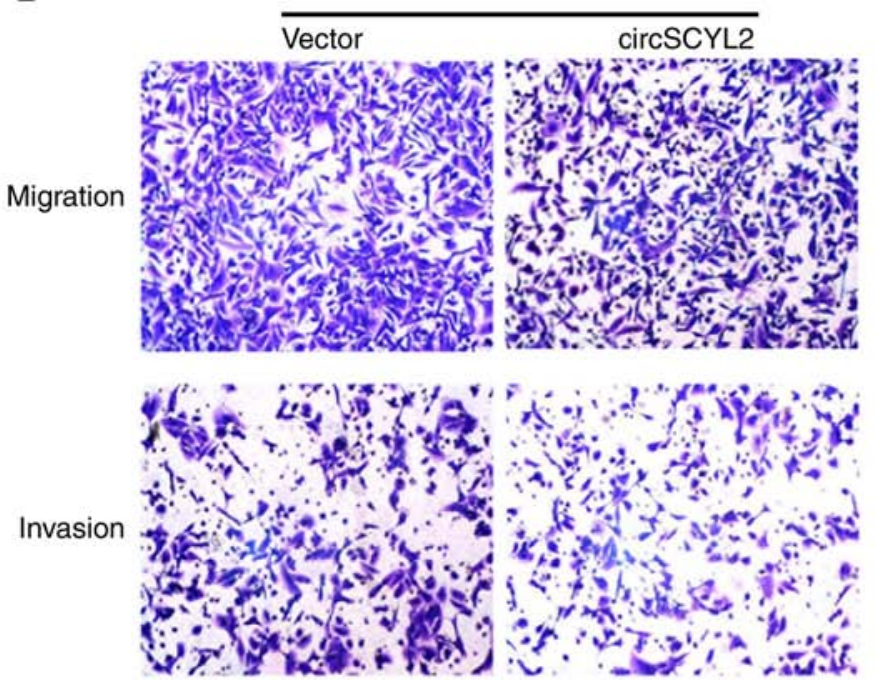

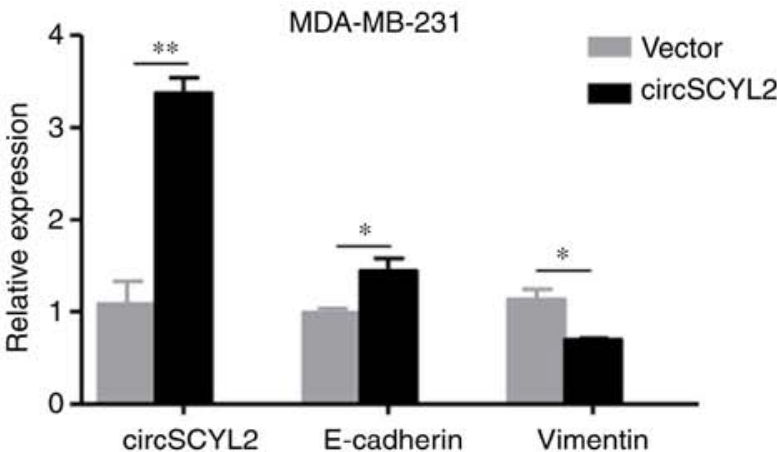

MDA-MB-231
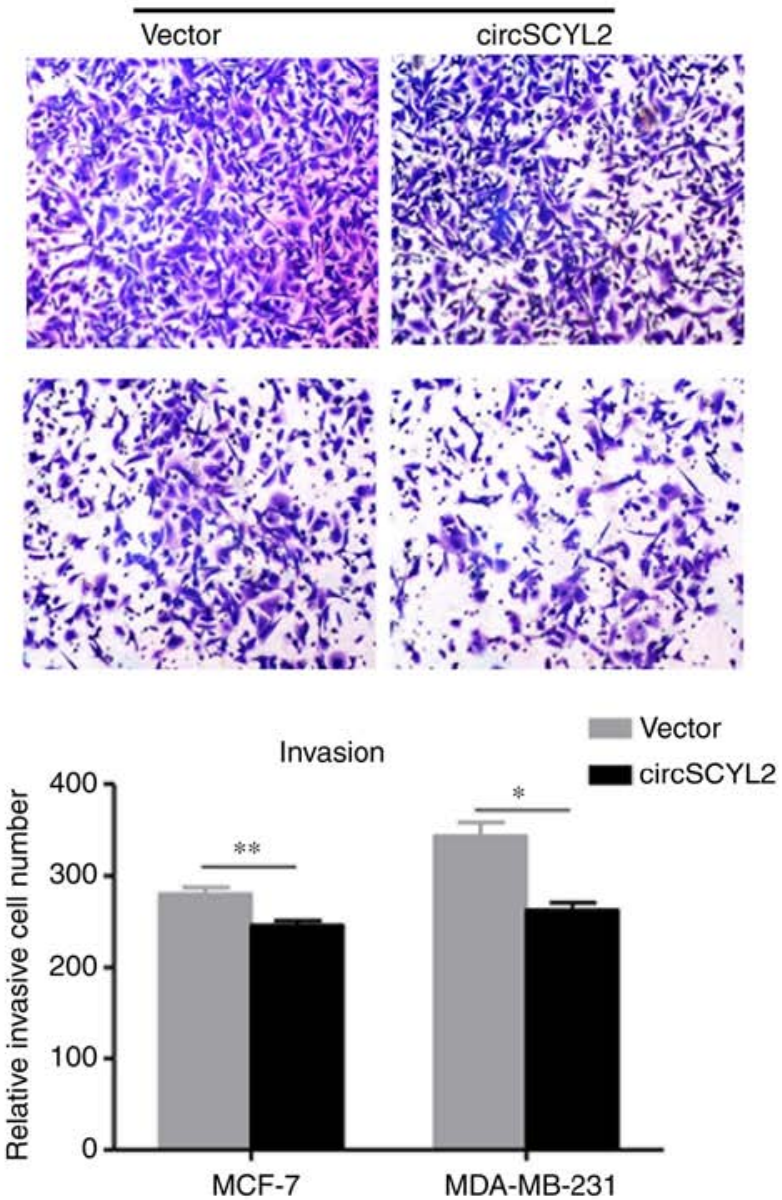

Figure 7. Over-expression of circSCYL2 inhibits the migration and invasion of breast cancer cells. (A) The expression levels of circSCYL2, E-cadherin and Vimentin in MCF-7 and MDA-MB-231 cells after transfection with circSCYL2 or control vector plasmids were detected by RT-qPCR. The value of $2^{-\Delta \Delta C q}$ was used to show the expressional level of RNAs. Data are expressed as mean \pm SD. ${ }^{*} \mathrm{P}<0.05$ and ${ }^{* *} \mathrm{P}<0.01$. (B) Cell migration and invasion abilities of MCF-7 and MDA-MB-231 cells transfected with circSCYL2 or the control vector were evaluated by transwell migration and matrigel invasion assays (magnification, $\mathrm{x} 100$ ). Data are mean $\pm \mathrm{SD}, \mathrm{n}=3 .{ }^{*} \mathrm{P}<0.05$ and ${ }^{* *} \mathrm{P}<0.01$. RT-q, reverse transcription-quantitative; circ, circular; E, epithelial.

migration and invasion (48). There was also some evidence that OSR1 is a target of TGF- $\beta$ signaling (49), which has a predominant role and its activation can lead to the expression of key transcription factors of EMT, such as SNAIL, ZEB and basic helix-loop-helix transcription factors (50). During the malignant phases of breast cancer progression, the TGF- $\beta$ signaling pathway elicits tumor promoting effects particularly by driving the EMT, which enhances tumor cell migration, invasion and ultimately metastasis to distant organs (51). The current study showed circSCYL2 and its target gene OSR1 targeting the TGF- $\beta$ signaling pathway were downregulated in the EMT model of breast cancer cells, which demonstrates that circSCYL2 may regulate EMT through OSR1/TGF- $\beta$ axis.

In conclusion, circRNAs expression profiles were identified and differentially expressed circRNAs were screened between EMT and the blank group of two breast cancer cells, and these circRNAs were associated with immune/inflammatory response and cell proliferation/cell adhesion functions by bioinformatic analysis. The present study found circSCYL2 and its target gene OSR1 were downregulated in breast cancer cells, and the overexpression of circSCYL2 in breast cancer 
cells inhibited the abilities of cell migration and invasion. All these suggest that further research of circRNAs related to EMT progression may provide a potential diagnostic and therapeutic biomarker for breast cancer. Therefore, the relationship of circRNAs was only inferred and more biological experiments are still needed to verify the interactions among them; the molecular mechanism underlying the association of circRNAs with tumor metastasis should be experimentally identified and characterized in the future.

\section{Acknowledgements}

Not applicable.

\section{Funding}

No funding was received.

\section{Availability of data and materials}

The datasets used and/or analyzed during the current study are available from the corresponding author on reasonable request.

\section{Authors' contributions}

SD was involved in the conceptualization of the study and in funding acquisition. $\mathrm{CY}, \mathrm{XL}, \mathrm{XZ}$ and $\mathrm{HZ}$ performed the experiments and were involved in the investigative aspects of the study. CY was involved in data curation. XL was involved in the study methodology. XZ and $\mathrm{HZ}$ were involved in the interpretation of the results and in the provision of software, and also created the figures. CY, XL and XZ were involved in the writing of the original draft and in the revision of the manuscript. All authors read and approved the final manuscript.

\section{Ethics approval and consent to participate}

The study was approved by the Ethics Committee of the Second Affiliated Hospital of Nanchang University. All patients provided written informed consent.

\section{Patient consent for publication}

Not applicable.

\section{Competing interests}

The authors declare that they have no competing interests.

\section{References}

1. Siegel RL, Miller KD and Jemal A: Cancer statistics, 2017. CA Cancer J Clin 67: 7-30, 2017.

2. Zhou J, Zhang WW, Peng F, Sun JY, He ZY and Wu SG: Downregulation of hsa_circ_0011946 suppresses the migration and invasion of the breast cancer cell line MCF-7 by targeting RFC3. Cancer Manag Res 10: 535-544, 2018.

3. Rodgers RJ, Reid GD, Koch J, Deans R, Ledger WL, Friedlander M, Gilchrist RB, Walters KA and Abbott JA: The safety and efficacy of controlled ovarian hyperstimulation for fertility preservation in women with early breast cancer: A systematic review. Hum Reprod 32: 1033-1045, 2017.
4. Zhou SY, Chen W, Yang SJ, Xu ZH, Hu JH, Zhang HD, Zhong SL and Tang JH: The emerging role of circular RNAs in breast cancer. Biosci Rep 39: BSR20190621, 2019.

5. Hattori $\mathrm{M}$ and Iwata $\mathrm{H}$ : Advances in treatment and care in metastatic breast cancer (MBC): Are there MBC patients who are curable? Chin Clin Oncol 7: 23, 2018.

6. Andersson Y, Bergkvist L, Frisell J and de Boniface J: Long-term breast cancer survival in relation to the metastatic tumor burden in axillary lymph nodes. Breast Cancer Res Treat 171: 359-369, 2018.

7. Wang Z, Katsaros D, Biglia N, Shen Y, Fu Y, Loo LWM, Jia W, Obata Y and Yu H: High expression of long non-coding RNA MALAT1 in breast cancer is associated with poor relapse-free survival. Breast Cancer Res Treat 171: 261-271, 2018.

8. SobierajskaK, Ciszewski WM, Wawro ME,Wieczorek-SzukałaK, Boncela J,Papiewska-Pajak I, Niewiarowska J and Kowalska MA: TUBB4B downregulation is critical for increasing migration of metastatic colon cancer cells. Cells 8: E810, 2019.

9. Arnold JM, Gu F, Ambati CR, Rasaily U, Ramirez-Pena E, Joseph R, Manikkam M, San Martin R, Charles C, Pan Y, et al: UDP-glucose 6-dehydrogenase regulates hyaluronic acid production and promotes breast cancer progression. Oncogene: July 15, 2019 (Epub ahead of print).

10. Gruenbacher $\mathrm{G}$ and Thurnher M: Mevalonate metabolism in cancer stemness and trained immunity. Front Oncol 8: 394, 2018.

11. Bhowmik SK, Ramirez-Peña E, Arnold JM, Putluri V, Sphyris N, Michailidis G, Putluri N, Ambs S, Sreekumar A and Mani SA: EMT-induced metabolite signature identifies poor clinical outcome. Oncotarget 6: 42651-42660, 2015.

12. Porshneva K, Papiernik D, Psurski M, Łupicka-Słowik A, Matkowski R, Ekiert M, Nowak M, Jarosz J, Banach J, Milczarek M, et al: Temporal inhibition of mouse mammary gland cancer metastasis by CORM-A1 and DETA/NO combination therapy. Theranostics 9: 3918-3939, 2019.

13. Wang HF, Wang SS, Zheng M, Dai LL, Wang K, Gao XL, Cao MX, Yu XH, Pang X, Zhang M, et al: Hypoxia promotes vasculogenic mimicry formation by vascular endothelial growth factor A mediating epithelial-mesenchymal transition in salivary adenoid cystic carcinoma. Cell Prolif 52: e12600, 2019.

14. Singh S and Chakrabarti R: Consequences of EMT-driven changes in the immune microenvironment of breast cancer and therapeutic response of cancer cells. J Clin Med 8: E642, 2019.

15. Yang HL, Thiyagarajan V, Shen PC, Mathew DC, Lin KY, Liao JW and Hseu YC: Anti-EMT properties of CoQ0 attributed to $\mathrm{PI} 3 \mathrm{~K} / \mathrm{AKT} / \mathrm{NFKB} / \mathrm{MMP}-9$ signaling pathway through ROS-mediated apoptosis. J Exp Clin Cancer Res 38: 186, 2019.

16. Gener P, Rafael D, Seras-Franzoso J, Perez A, Pindado LA, Casas G, Arango D, Fernández Y, Díaz-Riascos ZV, Abasolo I and Schwartz S Jr: Pivotal role of AKT2 during dynamic phenotypic change of breast cancer stem cells. Cancers (Basel) 11: E1058, 2019.

17. Holdt LM, Kohlmaier A and Teupser D: Molecular roles and function of circular RNAs in eukaryotic cells. Cell Mol Life Sci 75: 1071-1098, 2018.

18. Patop IL, Wüst $S$ and Kadener S: Past, present, and future of circRNAs. EMBO J 38: e100836, 2019.

19. Du WW, Yang W, Liu E, Yang Z, Dhaliwal P and Yang BB: Foxo3 circular RNA retards cell cycle progression via forming ternary complexes with p21 and CDK2. Nucleic Acids Res 44: 2846-2858, 2016.

20. Guarnerio J, Bezzi M, Jeong JC, Paffenholz SV, Berry K, Naldini MM, Lo-Coco F, Tay Y, Beck AH and Pandolfi PP: Oncogenic role of fusion-circRNAs derived from cancer-associated chromosomal translocations. Cell 165: 289-302, 2016.

21. Liang Y, Song X, Li Y, Ma T, Su P, Guo R, Chen B, Zhang H, Sang Y, Liu Y, et al: Targeting the circBMPR2/miR-553/USP4 axis as a potent therapeutic approach for breast cancer. Mol Ther Nucleic Acids 17: 347-361, 2019.

22. Yang L, Song C, Chen Y, Jing G and Sun J: Circular RNA circ_0103552 forecasts dismal prognosis and promotes breast cancer cell proliferation and invasion by sponging miR-1236. J Cell Biochem 120: 15553-15560, 2019.

23. Xu JH, Wang Y and Xu D: Hsa_circ_001569 is an unfavorable prognostic factor and promotes cell proliferation and metastasis by modulating PI3K-AKT pathway in breast cancer. Cancer Biomark 25: 193-201,2019.

24. Hatta M, Miyake Y, Uchida K and Yamazaki J: Keratin 13 gene is epigenetically suppressed during transforming growth factor-beta1-induced epithelial-mesenchymal transition in a human keratinocyte cell line. Biochem Biophys Res Commun 496: 381-386, 2018. 
25. Suzuki S, Toyoma S, Tsuji T, Kawasaki Y and Yamada T: CD147 mediates transforming growth factor- $\beta 1$-induced epithelial-mesenchymal transition and cell invasion in squamous cell carcinoma of the tongue. Exp Ther Med 17: 2855-2860, 2019.

26. Livak KJ and Schmittgen TD: Analysis of relative gene expression data using real-time quantitative PCR and the 2(-Delta Delta C(T)) method. Methods 25: 402-408, 2001.

27. Martínez-Reza I, Díaz L and García-Becerra R: Preclinical and clinical aspects of TNF- $\alpha$ and its receptors TNFR1 and TNFR2 in breast cancer. J Biomed Sci 24: 90, 2017.

28. Manna PR, Ahmed AU, Yang S, Narasimhan M, Cohen-Tannoudji J, Slominski AT and Pruitt K: Genomic profiling of the steroidogenic acute regulatory protein in breast cancer: In silico assessments and a mechanistic perspective. Cancers (Basel) 11: E623, 2019.

29. Saleh R, Taha RZ, Sasidharan Nair V, Alajez NM and Elkord E: PD-L1 blockade by atezolizumab downregulates signaling pathways associated with tumor growth, metastasis, and hypoxia in human triple negative breast cancer. Cancers (Basel) 11: E1050, 2019.

30. Piperigkou Z and Karamanos NK: Estrogen receptor-mediated targeting of the extracellular matrix network in cancer. Semin Cancer Biol: July 13, 2019 (Epub ahead of print).

31. Sharma S, Wu SY, Jimenez H, Xing F, Zhu D, Liu Y, Wu K, Tyagi A, Zhao D, Lo HW, et al: $\mathrm{Ca}^{2+}$ and CACNA1H mediate targeted suppression of breast cancer brain metastasis by AM RF EMF. EBioMedicine 44: 194-208, 2019.

32. Gao JL, Peng K, Shen MW, Hou YH, Qian XB, Meng XW, Ji FH, Wang LN and Yang JP: Suppression of WNK1-SPAK/OSR1 attenuates bone cancer pain by regulating $\mathrm{NKCC} 1$ and $\mathrm{KCC} 2$. J Pain 20: 1416-1428, 2019.

33. Prabhu VV, Madhukar NS, Gilvary C, Kline CLB, Oster S, El-Deiry WS, Elemento O, Doherty F, VanEngelenburg A, Durrant $\mathrm{J}$, et al: Dopamine receptor D5 is a modulator of tumor response to dopamine receptor D2 antagonism. Clin Cancer Res 25: 2305-2313, 2019.

34. Wu Q, Li J, Li Z, Sun S, Zhu S, Wang L, Wu J, Yuan J, Zhang Y, Sun $S$ and Wang C: Exosomes from the tumour-adipocyte interplay stimulate beige/brown differentiation and reprogram metabolism in stromal adipocytes to promote tumour progression. J Exp Clin Cancer Res 38: 223, 2019.

35. Chu H, Hui G, Yuan L, Shi D, Wang Y, Du M, Zhong D, Ma L, Tong N, Qin C, et al: Identification of novel piRNAs in bladder cancer. Cancer Lett 356: 561-567, 2015

36. Ma B, Wells A and Clark AM: The pan-therapeutic resistance of disseminated tumor cells: Role of phenotypic plasticity and the metastatic microenvironment. Semin Cancer Biol: July 31, 2019 (Epub ahead of print).

37. Tang H, Huang X, Wang J, Yang L, Kong Y, Gao G, Zhang L, Chen ZS and Xie X: circKIF4A acts as a prognostic factor and mediator to regulate the progression of triple-negative breast cancer. Mol Cancer 18: 23, 2019.

38. Ye F, Gao G, Zou Y, Zheng S, Zhang L, Ou X, Xie X and Tang $\mathrm{H}$ : circFBXW7 inhibits malignant progression by sponging miR-197-3p and encoding a 185-aa protein in triple-negative breast cancer. Mol Ther Nucleic Acids 18: 88-98, 2019.
39. Garikipati VNS, Verma SK, Cheng Z, Liang D, Truongcao MM, Cimini M, Yue Y, Huang G, Wang C, Benedict C, et al: Circular RNA CircFndc3b modulates cardiac repair after myocardial infarction via FUS/VEGF-A axis. Nat Commun 10: 4317, 2019.

40. Zhang Y, Huang R, Cheng M, Wang L, Chao J, Li J, Zheng P, Xie P, Zhang $\mathrm{Z}$ and Yao H: Gut microbiota from NLRP3-deficient mice ameliorates depressive-like behaviors by regulating astrocyte dysfunction via circHIPK2. Microbiome 7: 116, 2019.

41. Su Y, Feng W, Shi J, Chen L, Huang J and Lin T: circRIP2 accelerates bladder cancer progression via miR-1305/Tgf- $\beta 2 / \mathrm{smad} 3$ pathway. Mol Cancer 19: 23, 2020.

42. Chen X, Mao R, Su W, Yang X, Geng Q, Guo C, Wang Z, Wang J, Kresty LA, Beer DG, et al: Circular RNA circHIPK3 modulates autophagy via MIR124-3p-STAT3-PRKAA/AMPK $\alpha$ signaling in STK11 mutant lung cancer. Autophagy: 1-13, 2019.

43. Tian C, Tang X, Zhu X, Zhou Q, Guo Y, Zhao R, Wang D and Gong B: Expression profiles of circRNAs and the potential diagnostic value of serum circMARK3 in human acute stanford type A aortic dissection. PLoS One 14: e0219013, 2019.

44. Yao Y, Chen X, Yang H, Chen W, Qian Y, Yan Z, Liao T, Yao W, Wu W, Yu T, et al: Hsa_circ_0058124 promotes papillary thyroid cancer tumorigenesis and invasiveness through the NOTCH3/GATAD2A axis. J Exp Clin Cancer Res 38: 318, 2019.

45. Liang M, Huang G, Liu Z, Wang Q, Yu Z, Liu Z, Lin H, Li M, Zhou X and Zheng Y: Elevated levels of hsa_circ_006100 in gastric cancer promote cell growth and metastasis via miR-195/GPRC5A signalling. Cell Prolif 52: e12661, 2019.

46. Zhang Y, Yuan Y, Liang P, Guo X, Ying Y, Shu XS, Gao M Jr and Cheng Y: OSR1 is a novel epigenetic silenced tumor suppressor regulating invasion and proliferation in renal cell carcinoma. Oncotarget 8: 30008-30018, 2017.

47. Otani K, Dong Y, Li X, Lu J, Zhang N, Xu L, Go MY, Ng EK, Arakawa T, Chan FK, et al: Odd-skipped related 1 is a novel tumour suppressor gene and a potential prognostic biomarker in gastric cancer. J Pathol 234: 302-315, 2014

48. Chen W, Wu K, Zhang H, Fu X, Yao F and Yang A: Odd-skipped related transcription factor 1 (OSR1) suppresses tongue squamous cell carcinoma migration and invasion through inhibiting NF- $\kappa \mathrm{B}$ pathway. Eur J Pharmacol 839: 33-39, 2018.

49. Gallolu Kankanamalage S, Karra AS and Cobb MH: WNK pathways in cancer signaling networks. Cell Commun Signal 16: 72, 2018.

50. Liao JY, Wu J, Wang YJ, He JH, Deng WX, Hu K, Zhang YC, Zhang Y, Yan H, Wang DL, et al: Deep sequencing reveals a global reprogramming of lncRNA transcriptome during EMT. Biochim Biophys Acta Mol Cell Res 1864: 1703-1713, 2017.

51. Suriyamurthy S, Baker D, Ten Dijke P and Iyengar PV: Epigenetic reprogramming of TGF- $\beta$ signaling in breast cancer. Cancers (Basel) 11: E726, 2019.

This work is licensed under a Creative Commons Attribution-NonCommercial-NoDerivatives 4.0 International (CC BY-NC-ND 4.0) License. 